\title{
Phytoplankton composition of the Itaparica and Xingó reservoirs, São Francisco River, Brazil
}

\author{
Aragão-Tavares, NKC. ${ }^{a}$, Severiano, JS. ${ }^{a}$ and Moura, AN. ${ }^{a *}$ \\ aPrograma de Pós-Graduação em Botânica - PPGB, Departamento de Biologia, Universidade Federal \\ Rural de Pernambuco - UFRPE, Rua Dom Manoel de Medeiros, s/n, Dois Irmãos, CEP 52171-900, Recife, PE, Brazil \\ *e-mail: ariadne_moura@hotmail.com
}

Received: October 15, 2013 - Accepted: May 14, 2014 - Distributed: August 31, 2015

(With 3 figures)

\begin{abstract}
The installation of dams causes changes to the integrity of rivers and to the water cycle, performing an instrumental role in the organization of biological communities, including that of phytoplankton. In the present study, we analyzed the taxonomic composition of phytoplankton in two hydroelectric reservoirs on the São Francisco River, Itaparica and Xingó reservoirs. Samples were collected at quarterly intervals between December 2007 and September 2009, at 12 sampling stations in each reservoir, totaling 92 samples. We identified 110 species in the Itaparica reservoir and 136 in the Xingó reservoir, of which diatoms followed by green algae, played a major contribution to both reservoirs. Most of the species is rare and/or occasional. In the Itaparica reservoir, there were no very frequent species, although in the Xingó this category was represented by the diatoms Aulacoseira granulata (Ehrenberg) Simonsen and Fragilaria crotonensis Kitton. These results show that, despite the similarity in the composition of phytoplankton, the reservoirs studied certainly differed regarding their environmental conditions.
\end{abstract}

Keywords: check list, freshwater, hydroelectric reservoir, microalgae, Northeast.

\section{Composição fitoplanctônica dos reservatórios de Itaparica e Xingó, Rio São Francisco, Brasil}

\begin{abstract}
Resumo
A instalação de barragens provoca alterações na integridade dos rios e no ciclo hidrológico, desempenhando papel decisivo na organização das comunidades biológicas, incluindo a fitoplanctônica. No presente estudo, foi realizada uma análise da composição taxonômica do fitoplâncton em dois reservatórios hidrelétricos do rio São Francisco, reservatórios Itaparica e Xingó. As coletas foram realizadas entre dezembro de 2007 e setembro de 2009, com intervalos trimestrais, em 12 estações de amostragem em cada reservatório, totalizando 192 amostras. Foram identificadas 110 espécies no reservatório de Itaparica e 136 no reservatório de Xingó, sendo observado, em ambos, maior contribuição das diatomáceas, seguidas pelas algas verdes. A maioria das espécies foi considerada esporádica e/ou pouco frequente. No reservatório de Itaparica, não foram registradas espécies muito frequentes, já no reservatório de Xingó esta categoria esteve representada pelas diatomáceas Aulacoseira granulata (Ehrenberg) Simonsen e Fragilaria crotonensis Kitton. Esses resultados mostram que, apesar da semelhança na composição fitoplanctônica, os reservatórios estudados, certamente, diferem nas condições ambientais.
\end{abstract}

Palavras-chave: check list, água doce, reservatório hidrelétrico, microalgas, Nordeste.

\section{Introduction}

The impacts of hydroelectric reservoirs on aquatic environments have been extensively studied (Kelly, 2001; Tundisi and Matsumura-Tundisi, 2003; Li et al., 2012), since the installation of dams causes changes to the integrity of Rivers and to the water cycle, playing an instrumental role in the organization of biological communities (Tundisi et al., 2002; Pringle, 2003; Silva and Cecy, 2004; Magilligan and Nislow, 2005).

Among the communities that best express the behavior of a water body is phytoplankton community, since they respond quickly to changing environmental conditions, both of natural and anthropogenic origin (Chellappa et al., 2009; Popovskaya et al., 2012). Thus, knowledge about the diversity and distribution of phytoplankton in water bodies is of fundamental importance for a better understanding of the functioning mechanisms of these ecosystems, possibly 
acquiring a predictive character about the possible changes that may occur in the environment (Huszar et al., 2000).

The São Francisco River, located in the main hydrographic basin of the semiarid region of Brazil, has seven dams along its course, built for electric power generation (ANA, 2013). The present study was conducted for two consecutive years, and two reservoirs (Xingó and Itaparica) were monitored in order to characterize the phytoplankton community in these environments, through the analysis of taxonomic composition, an important tool commonly used in monitoring programs to assess water quality worldwide.

\section{Material and Methods}

\subsection{Study area}

The São Francisco River has an extension of $2.700 \mathrm{~km}$ and its basin is the third largest in Brazil, with an area of $638.576 \mathrm{~km}^{2}$, accounting for $8 \%$ of the national territory. It extends through the Northeast, Southeast and Central West regions, including the Pernambuco, Alagoas, Sergipe, Bahia, Minas Gerais, Goiás States and the Federal District. It is divided into four physiographic regions (ANA, 2013) (Figure 1): Upper São Francisco, from the source to the municipality of Pirapora (Minas Gerais State), with an extension of 630 km; Middle São Francisco, from Pirapora to the municipality of Remanso (Bahia State) with an extension of 1090 km; Sub-Middle São Francisco, from Remanso to Paulo Afonso Falls (state of Bahia), with an extension of 686 km; Lower São Francisco, from Paulo Afonso Falls to the mouth in the Atlantic Ocean, on the border between the Alagoas and Sergipe State, with an extension of $274 \mathrm{~km}$.

Itaparica reservoir $\left(9^{\circ} 6^{\prime} \mathrm{S}\right.$ and $\left.38^{\circ} 19^{\prime} \mathrm{W}\right)$, belonging to the Luiz Gonzaga Hydroelectric Plant, is located on the Sub-Middle São Francisco, on the border between the Pernambuco and Bahia State, having an accumulated storage capacity of $10^{9} \mathrm{~m}^{3}$. Xingó reservoir $\left(9^{\circ} 37^{\prime} \mathrm{S}\right.$ and $37^{\circ} 47^{\prime} \mathrm{W}$ ) is located on the Lower São Francisco, on the border between the Alagoas and Sergipe States, and has an accumulated storage capacity of $3.8^{8} \mathrm{~m}^{3}$ (Brasil, 2013).

\subsection{Data collection and analysis}

Samples were collected at quarterly intervals over two years, from December 2007 to September 2009, at 12 stations located along the longitudinal axis of each reservoir (Figure 1, Table 1). Samples were obtained by vertical hauls of the net along the euphotic zone, determined by a digital quantometer (Licor-250), using conical-cylindrical plankton nets with a $25 \mu \mathrm{m}$ mesh size. The material was preserved in $4 \%$ formaldehyde and then examined using an optical microscope and photographed with the aid of a microscope (Zeiss/Axioskop) coupled to a Samsung SCC833, Japan camera, using the software Imagelab (Softium, Brazil).

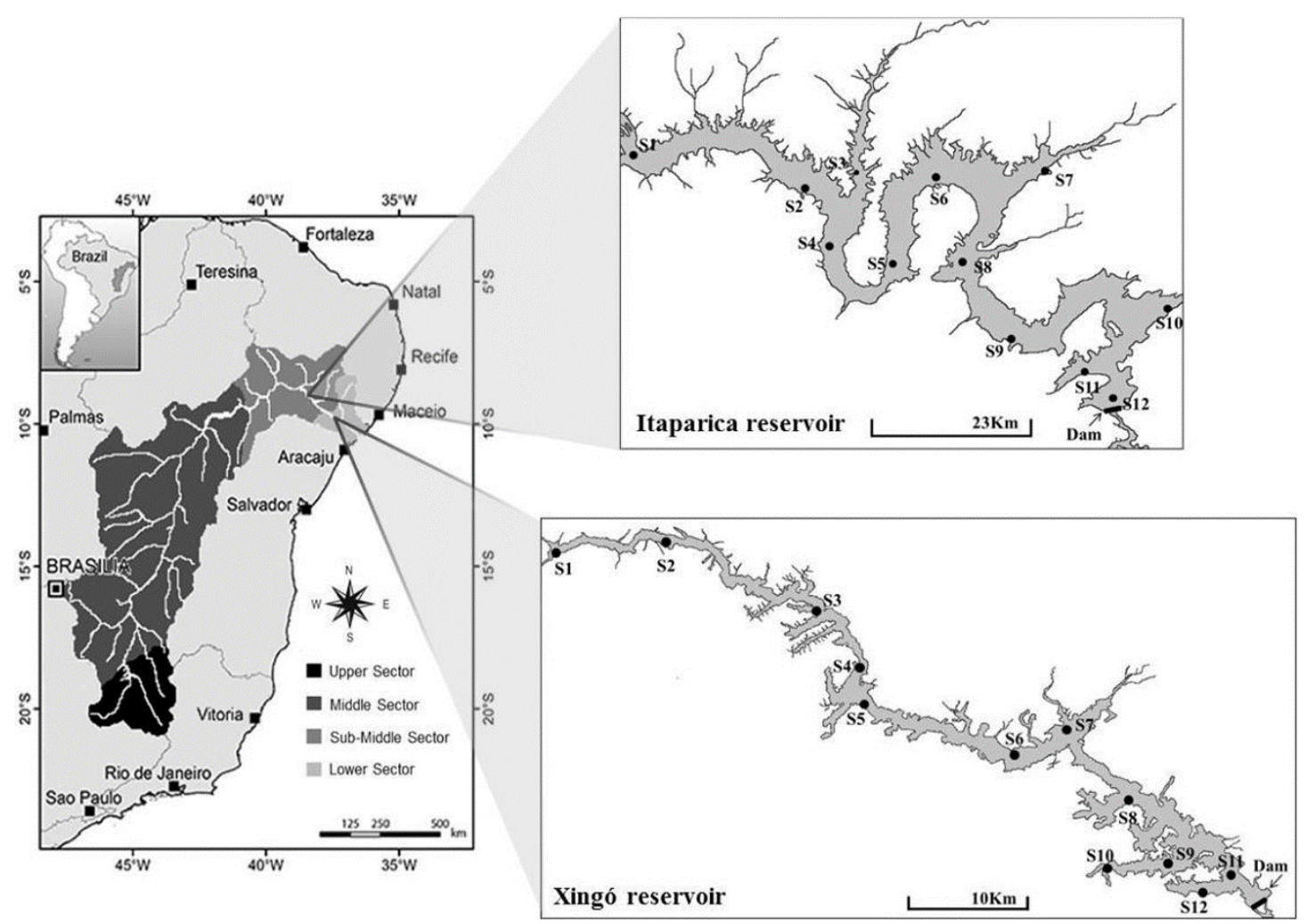

Figure 1. Location of the Itaparica and Xingó Reservoirs, São Francisco River, Brazil. 
Aragão-Tavares, NKC., Severiano, JS. and Moura, AN.

Table 1. Geographic location of the sampling stations in the Itaparica and Xingó Reservoirs, São Francisco River, Brazil.

\begin{tabular}{|c|c|c|c|c|}
\hline \multirow{3}{*}{$\begin{array}{c}\text { Stations } \\
1\end{array}$} & \multicolumn{4}{|c|}{ Reservoirs } \\
\hline & \multicolumn{2}{|c|}{ Itaparica } & \multicolumn{2}{|c|}{ Xingó } \\
\hline & $8^{\circ} 47^{\prime} 52.6^{\prime \prime} \mathrm{S}$ & $38^{\circ} 57^{\prime} 71.9^{\prime \prime} \mathrm{W}$ & $9^{\circ} 26^{\prime} 26.09^{\prime \prime} \mathrm{S}$ & $38^{\circ} 09^{\prime} 18.11^{\prime \prime} \mathrm{W}$ \\
\hline 2 & $8^{\circ} 50^{\prime} 18.6 ” \mathrm{~S}$ & $38^{\circ} 43^{\prime} 65.5 ” \mathrm{~W}$ & $9^{\circ} 26^{\prime} 13.16^{\prime \prime} \mathrm{S}$ & $38^{\circ} 05^{\prime} 51.72 ” \mathrm{~W}$ \\
\hline 3 & $8^{\circ} 49^{\prime} 23.1$ ' S & $38^{\circ} 39^{\prime} 44.3$ ' W & $9^{\circ} 28^{\prime} 16.57 ” \mathrm{~S}$ & $38^{\circ} 01^{\prime} 20.96 ” \mathrm{~W}$ \\
\hline 4 & $8^{\circ} 54 ’ 37.3 ” \mathrm{~S}$ & $38^{\circ} 41^{\prime} 10.4$ ' W & $9^{\circ} 29^{\prime} 57.41^{\prime \prime} \mathrm{S}$ & $37^{\circ} 59^{\prime} 58.52^{\prime} \mathrm{W}$ \\
\hline 5 & $8^{\circ} 55^{\prime} 73.7^{\prime \prime} \mathrm{S}$ & $38^{\circ} 36^{\prime} 34.2^{\prime \prime} \mathrm{W}$ & $9^{\circ} 31^{\prime} 33.24$ ' S & $37^{\circ} 59^{\prime} 22.24^{\prime \prime} \mathrm{W}$ \\
\hline 6 & $8^{\circ} 48^{\prime} 72.0^{\prime \prime} \mathrm{S}$ & $38^{\circ} 33^{\prime} 25.2^{\prime \prime} \mathrm{W}$ & $9^{\circ} 32 ’ 44.05 \% \mathrm{~S}$ & $37^{\circ} 55^{\prime} 09.52^{\prime} \mathrm{W}$ \\
\hline 7 & $8^{\circ} 49^{\prime} 23.1$ ' S & $38^{\circ} 25^{\prime} 21.5^{\prime \prime} \mathrm{W}$ & $9^{\circ} 31^{\prime} 40.333^{\prime \prime} \mathrm{S}$ & $37^{\circ} 53 ’ 22.81^{\prime \prime} \mathrm{W}$ \\
\hline 8 & $8^{\circ} 55^{\prime} 99.6 ” \mathrm{~S}$ & $38^{\circ} 31^{\prime} 02.3 " \mathrm{~W}$ & 9॰34'06.96” S & $37^{\circ} 51^{\prime} 43.63^{\prime \prime} \mathrm{W}$ \\
\hline 9 & $9^{\circ} 01^{\prime} 96.0^{\prime \prime} \mathrm{S}$ & $38^{\circ} 27^{\prime} 13.3 ” \mathrm{~W}$ & 9॰36’01.94” S & $37^{\circ} 50^{\prime} 35.81^{\prime \prime} \mathrm{W}$ \\
\hline 10 & $8^{\circ} 59 ' 81.7 ” \mathrm{~S}$ & $38^{\circ} 14^{\prime} 17.0 ” \mathrm{~W}$ & $9^{\circ} 36^{\prime} 22.57^{\prime \prime} \mathrm{S}$ & $37^{\circ} 52^{\prime} 48.64^{\prime \prime} \mathrm{W}$ \\
\hline 11 & $9^{\circ} 05^{\prime} 06.0^{\prime \prime} \mathrm{S}$ & $38^{\circ} 21^{\prime} 26.1^{\prime \prime} \mathrm{W}$ & $9^{\circ} 36^{\prime} 24.91 ” S$ & $37^{\circ} 52^{\prime} 47.78^{\prime \prime} \mathrm{W}$ \\
\hline 12 & $9^{\circ} 07^{\prime} 72.8^{\prime \prime} \mathrm{S}$ & $38^{\circ} 18^{\prime} 57.7^{\prime \prime} \mathrm{W}$ & $9^{\circ} 36^{\prime} 54.18^{\prime \prime} \mathrm{S}$ & $37^{\circ} 48^{\prime} 03.96 " \mathrm{~W}$ \\
\hline
\end{tabular}

Permanent slides for identifying diatoms were prepared following the methodology proposed by Carr et al. (1986), using Naphrax.

The classification system adopted followed Van den Hoek et al. (1995) for the classes Cryptophyceae, Dinophyceae, Chrysophyceae, Euglenophyceae, Chlorophyceae and Zygnematophyceae. Round et al. (1990) was used For Coscinodiscophyceae, Fragilariophyceae and Bacillariophyceae, while for Cyanobacteria, Komárek and Anagnostidis $(2000,2005)$ were used. Following the taxonomic analysis and photomicrographs, samples were deposited in the Herbarium Professor Vasconcelos Sobrinho, Federal Rural University of Pernambuco.

The frequency of occurrence of taxa was calculated according to Matteucci and Colma (1982), considering the number of samples in which the taxon occurred in relation to the total number of samples collected, using the formula: $\mathrm{F}=\mathrm{Px} 100 / \mathrm{p}$, where $\mathrm{P}=$ number of samples in which the taxon was recorded and $p=$ total number of samples collected. The following criteria were established: very frequent $(\geq 70 \%)$, frequent $(\geq 40 \%<70 \%)$, occasional $(\geq 10 \%<40 \%)$ and rare $(<10 \%)$.

\section{Results and Discussion}

The phytoplankton community was made up of 165 species belonging to 10 classes, 28 orders, 49 families and 83 genera (Table 2). One hundred and ten species were recorded in the Itaparica reservoir and 135 in the Xingó reservoir (Table 2). In both reservoirs, diatoms (Coscinodiscophyceae, Bacillariophyceae and Fragilariophyceae) had the highest number of species, followed by green algae (Chlorophyceae and Zygnematophyceae) and cyanobacteria (Cyanobacteria) (Figure 2, Table 2).

This number of identified species is considered high when compared to that observed by Franca and Coimbra (1998), who recorded 54 species in the Itaparica reservoir, and Melo-Magalhães et al. (2000), who identified 78 species in the Xingó reservoir. However, this is compatible to that observed in other reservoirs used for power generation
(Pérez et al., 1999; Calijuri et al., 2002; Atici and Obali, 2006; Nogueira et al., 2010; Popovskaya et al., 2012). Certainly, the greater sampling effort exerted in this study, compared to that of Franca and Coimbra (1998) and Melo-Magalhães et al. (2000), made it possible to evaluate the true phytoplankton biodiversity in the studied environments.

The dominance, in terms of richness, of diatoms and green algae is considered almost standard in phytoplankton communities of most reservoirs in temperate (Pérez et al., 1999; Atici, 2002; Atici and Obali, 2006; Malaiwan and Peerapornpisal, 2009; Popovskaya et al., 2012), tropical (De León and Chalar, 2003) and subtropical (Çetin and Şen, 1998) regions. In Brazil, the same result was observed in the reservoirs Jurumirim and Bonita in the São Paulo State (Nogueira, 2000; Calijuri et al., 2002); the reservoirs Capivara, Foz do Areia, Salto Caxias, Salto Osório, Salto Santiago and Segredo, in the Paraná State (Silva et al., 2005; Borges et al., 2008); and in the Cachoeira Dourada reservoir, in the states of Goiás/Minas Gerais (Oliveira et al., 2011).

The success of diatoms and green algae in these aquatic ecosystems has been associated to the great morphological and habitat diversity presented by the species (Wetzel, 1993; Franceschini et al., 2010). Diatoms are widely distributed in nature, occurring in all aquatic ecosystems, although they are more abundant in the marine environment (Van den Hoek et al., 1995; Reviers, 2006). Currently, there are about 250 genera and approximately 100,000 described species, 1,600 of which are found in freshwater environments. Green algae are a very diverse group, consisting of about 8,000 species, approximately $90 \%$ of which are found in freshwater environments (Van den Hoek et al., 1995).

Both groups are represented mostly by plankton species, although many are periphytic (Fontana and Bicudo, 2009; Felisberto and Rodrigues, 2010) or are associated with the bottom, living in the sediment, as in the case of diatoms (Silva et al., 2010). Climatological and hydrological events that promote turbulence in the system play an important role in the dynamics of these populations and in the increase in the number of phytoplanktonic species, since they can 
Table 2. Synopsis and frequency of occurrence (\%) of phytoplankton in the Itaparica and Xingó Reservoirs, São Francisco River, Brazil, between December 2007 and September 2008.

\begin{tabular}{|c|c|c|}
\hline & \multicolumn{2}{|c|}{ Reservoirs } \\
\hline 1 axa & Itaparica & Xingó \\
\hline \multicolumn{3}{|l|}{ Cyanophyceae } \\
\hline \multicolumn{3}{|l|}{ Order: Chroococcales } \\
\hline \multicolumn{3}{|l|}{ Family: Merismopediaceae } \\
\hline Aphanocapsa elachista W. West \& G.S. West & 11.5 & 16.7 \\
\hline Aphanocapsa incerta (Lemmermann) Cronberg \& Komárek & - & 4.4 \\
\hline Aphanocapsa sp. & 5.2 & 11.1 \\
\hline Merismopedia sp. & 2.1 & - \\
\hline \multicolumn{3}{|l|}{ Family: Microcystaceae } \\
\hline Microcystis aeruginosa (Kützing) Kützing & 2.1 & 3.3 \\
\hline $\begin{array}{l}\text { Microcystis panniformis J. Komárek. J. Komárková-Legnerová. C.L. Sant’Anna. } \\
\text { M.T.P. Azevedo. \& P.A.C. Senna }\end{array}$ & - & 1.1 \\
\hline Microcystis wesenbergii (Komárek) Komárek & 24.0 & 16.7 \\
\hline Microcystis sp. & 12.5 & 4.4 \\
\hline \multicolumn{3}{|l|}{ Family: Chroococcaceae } \\
\hline Chroococcus limneticus Lemmermann & 1.0 & - \\
\hline Chroococcus minutus (Kützing) Nägeli & - & 3.3 \\
\hline Chroococcus turgidus (Kützing) Nägeli & 2.1 & 8.9 \\
\hline Chroococcus sp. & - & 2.2 \\
\hline Cyanosarcina burmensis (Skuja) Kovácik & - & 3.3 \\
\hline \multicolumn{3}{|l|}{ Order: Oscillatoriales } \\
\hline \multicolumn{3}{|l|}{ Family: Oscillatoriaceae } \\
\hline Lyngbya sp. & 2.1 & 1.1 \\
\hline Oscillatoria princeps Vaucher ex Gomont & - & 5.6 \\
\hline Oscillatoria sancta Kützing ex Gomont & 1.0 & 3.3 \\
\hline Oscillatoria sp. & 34.4 & 11.1 \\
\hline \multicolumn{3}{|l|}{ Family: Pseudanabaenaceae } \\
\hline Geitlerinema amphibium (C. Agardh) Anagnostidis & 27.1 & 42.2 \\
\hline Geitlerinema splendidum (Greville) Anagnostidis & - & 1.1 \\
\hline Geitlerinema sp. & 9.4 & - \\
\hline Pseudanabaena catenata Lauterborn & 4.2 & 1.1 \\
\hline Pseudanabaena limnetica (Lemmermann) Komárek & - & 2.2 \\
\hline Pseudanabaena sp. & 3.1 & 2.2 \\
\hline \multicolumn{3}{|l|}{ Family: Phormidiaceae } \\
\hline Phormidium sp. & 3.1 & 4.4 \\
\hline Planktothrix agardhii (Gomont) Anagnostidis \& Komárek & 24.0 & 16.7 \\
\hline \multicolumn{3}{|l|}{ Order: Nostocales } \\
\hline \multicolumn{3}{|l|}{ Family: Nostocaceae } \\
\hline Anabaena circinalis Rabenhorst & 35.4 & 33.3 \\
\hline Anabaena constricta (Szafer) Geitler & 26.0 & 15.6 \\
\hline Anabaena sp. 1 & 14.6 & 16.7 \\
\hline Anabaena sp. 2 & - & 3.3 \\
\hline Anabaenopsis sp. & - & 1.1 \\
\hline Aphanizomenon sp. & 3.1 & 4.4 \\
\hline Cylindrospermopsis raciborskii (Woloszynska) Seenaya \& Subba Raju & 41.7 & 56.7 \\
\hline \multicolumn{3}{|l|}{ Coscinodiscophyceae } \\
\hline \multicolumn{3}{|l|}{ Order: Thalassiosirales } \\
\hline \multicolumn{3}{|l|}{ Family: Stephanodiscaceae } \\
\hline Cyclotella meneghiniana Kützing & - & 4.4 \\
\hline Cyclotella stelligera Cleve \& Grunow & - & 1.1 \\
\hline
\end{tabular}

- = absence of species. 
Table 2. Continued...

\begin{tabular}{|c|c|c|}
\hline \multirow{2}{*}{ Taxa } & \multicolumn{2}{|c|}{ Reservoirs } \\
\hline & Itaparica & Xingó \\
\hline \multicolumn{3}{|l|}{ Order: Melosirales } \\
\hline \multicolumn{3}{|l|}{ Family: Melosiraceae } \\
\hline Melosira varians C. Agardh & - & 1.1 \\
\hline \multicolumn{3}{|l|}{ Order: Aulacoseirales } \\
\hline \multicolumn{3}{|l|}{ Family: Aulacoseiraceae } \\
\hline Aulacoseira ambigua (Grunow) Simonsen & 49.0 & 50.0 \\
\hline Aulacoseira granulata (Ehrenberg) Simonsen & 56.3 & 72.2 \\
\hline Aulacoseira granulata var. angustissima (O.F. Müller) Simonsen & - & 2.2 \\
\hline Aulacoseira herzogii (Lemmermann) Simonsen & - & 1.1 \\
\hline \multicolumn{3}{|l|}{ Order: Triceratiales } \\
\hline \multicolumn{3}{|l|}{ Family: Triceratiaceae } \\
\hline Pleurosira laevis (Ehrenberg) Compère & 1.0 & - \\
\hline \multicolumn{3}{|l|}{ Order: Rhizosoleniales } \\
\hline \multicolumn{3}{|l|}{ Family: Rhizosoleniaceae } \\
\hline Urosolenia eriensis (H.L. Smith) F.E. Round \& R.M. Crawford & 4.2 & 12.2 \\
\hline Urosolenia longiseta (Zacharias) Bukhtiyarova & 39.6 & 42.2 \\
\hline \multicolumn{3}{|l|}{ Fragilariophyceae } \\
\hline \multicolumn{3}{|l|}{ Order: Fragilariales } \\
\hline \multicolumn{3}{|l|}{ Family: Fragilariaceae } \\
\hline Fragilaria crotonensis Kitton & - & 80.0 \\
\hline Fragilaria capucina Desmazières & 1.0 & - \\
\hline Fragilaria capucina var. fragilarioides (Grun.) Ludwig et Flores & - & 3.3 \\
\hline Fragilaria sp. & 7.3 & 13.3 \\
\hline Synedra acus Kützing & - & 1.1 \\
\hline Synedra sp. & - & 1.1 \\
\hline Ulnaria ulna (Nitzsch) P. Compère & 14.6 & 14.4 \\
\hline \multicolumn{3}{|l|}{ Bacillariophyceae } \\
\hline \multicolumn{3}{|l|}{ Order: Eunotiales } \\
\hline \multicolumn{3}{|l|}{ Family: Eunotiaceae } \\
\hline Eunotia sp. & 1.0 & 1.1 \\
\hline \multicolumn{3}{|l|}{ Order: Cymbellales } \\
\hline \multicolumn{3}{|l|}{ Family: Cymbellaceae } \\
\hline Encyonema silesiacum (Bleisch) D.G.Mann in Round. Crawford \& Mann & 1.0 & - \\
\hline Encyonema sp. & 1.0 & - \\
\hline \multicolumn{3}{|l|}{ Family: Gomphonemataceae } \\
\hline Gomphonema parvulum (Kützing) Grunow & - & 2.2 \\
\hline Gomphonema sp. & 2.1 & 1.1 \\
\hline \multicolumn{3}{|l|}{ Order: Naviculales } \\
\hline \multicolumn{3}{|l|}{ Family: Stauroneidaceae } \\
\hline Stauroneis phoenicenteron (Nitzsch) Ehrenberg & - & 1.1 \\
\hline \multicolumn{3}{|l|}{ Family: Pinnulariaceae } \\
\hline Pinnularia maior (Kützing) Cleve & 1.0 & 1.1 \\
\hline Pinnularia sp. & 9.4 & 2.2 \\
\hline Family: Amphipleuraceae & & \\
\hline Amphipleura pellucida (Kützing) Kützing & 1.0 & - \\
\hline Frustulia rhomboides (Ehrenberg) De Toni & 3.1 & - \\
\hline Family: Naviculaceae & & \\
\hline Navicula sp. & 4.2 & 5.6 \\
\hline Family: Pleurosigmataceae & & \\
\hline
\end{tabular}

- = absence of species. 
Table 2. Continued...

\begin{tabular}{|c|c|c|}
\hline \multirow{2}{*}{ Taxa } & \multicolumn{2}{|c|}{ Reservoirs } \\
\hline & Itaparica & Xingó \\
\hline Gyrosigma spenceri (W. Smith) Griffith \& Henfrey & 2.1 & 1.1 \\
\hline \multicolumn{3}{|l|}{ Order: Bacillariales } \\
\hline \multicolumn{3}{|l|}{ Family: Bacillariaceae } \\
\hline Nitzschia paleaeformis Hust & - & 2.2 \\
\hline \multicolumn{3}{|l|}{ Order: Rhopalodiales } \\
\hline \multicolumn{3}{|l|}{ Family: Rhopalodiaceae } \\
\hline Epithemia sorex Kützing & 2.1 & 3.3 \\
\hline Epithemia sp. & 1.0 & 1.1 \\
\hline \multicolumn{3}{|l|}{ Order: Surirellales } \\
\hline \multicolumn{3}{|l|}{ Family: Surirellaceae } \\
\hline Surirella robusta Ehrenberg & 7.3 & - \\
\hline Surirella sp. & 2.1 & 2.2 \\
\hline \multicolumn{3}{|l|}{ Chrysophyceae } \\
\hline \multicolumn{3}{|l|}{ Order: Monosigales } \\
\hline \multicolumn{3}{|l|}{ Family: Synuraceae } \\
\hline Mallomonas caudata Ivanov & - & 1.1 \\
\hline \multicolumn{3}{|l|}{ Order: Ochromonadales } \\
\hline \multicolumn{3}{|l|}{ Family: Dinobryaceae } \\
\hline Dinobryon sertularia Ehrenberg & 10.4 & 8.9 \\
\hline \multicolumn{3}{|l|}{ Cryptophyceae } \\
\hline \multicolumn{3}{|l|}{ Order: Cryptomonadales } \\
\hline \multicolumn{3}{|l|}{ Family: Cryptomonadaceae } \\
\hline Cryptomonas ovata Ehrenberg & 5.2 & 10.0 \\
\hline Cryptomonas subovalis Ehrenberg & 2.1 & 4.4 \\
\hline Cryptomonas sp. & - & 3.3 \\
\hline \multicolumn{3}{|l|}{ Order: Pyrenomonadales } \\
\hline \multicolumn{3}{|l|}{ Family: Pyrenomodaceae } \\
\hline Rhodomonas lacustris Pascher \& Ruttner & 1.0 & 3.3 \\
\hline Rhodomonas sp. & 1.0 & - \\
\hline \multicolumn{3}{|l|}{ Dinophyceae } \\
\hline \multicolumn{3}{|l|}{ Order: Peridiniales } \\
\hline \multicolumn{3}{|l|}{ Family: Gymnodiniaceae } \\
\hline Gymnodinium sp. & - & 1.1 \\
\hline \multicolumn{3}{|l|}{ Family: Peridiniaceae } \\
\hline Peridinium cinctum (O.F. Müller) Ehrenberg & - & 11.1 \\
\hline Peridinium sp. & 5.2 & 8.9 \\
\hline \multicolumn{3}{|l|}{ Euglenophyceae } \\
\hline Order: Euglenales & & \\
\hline Family: Euglenaceae & & \\
\hline Euglena acus Ehrenberg & - & 1.1 \\
\hline Euglena oxyuris Schmarda & - & 1.1 \\
\hline Euglena sp. & 2.1 & 4.4 \\
\hline Phacus longicauda (Ehrenberg) Dujardin & 1.0 & 1.1 \\
\hline Trachelomonas armata (Ehrenberg) F.Stein & 2.1 & \\
\hline Trachelomonas obesa Ehrenberg & - & 7.8 \\
\hline Trachelomonas oblonga Ehrenberg & 3.1 & 3.3 \\
\hline Trachelomonas volvocina Ehrenberg & 7.3 & 12.2 \\
\hline Trachelomonas sp. & 10.4 & 6.7 \\
\hline
\end{tabular}

- = absence of species. 
Table 2. Continued...

\begin{tabular}{|c|c|c|}
\hline \multirow{2}{*}{ Taxa } & \multicolumn{2}{|c|}{ Reservoirs } \\
\hline & Itaparica & Xingó \\
\hline \multicolumn{3}{|l|}{ Chlorophyceae } \\
\hline \multicolumn{3}{|l|}{ Order: Sphaeropleales } \\
\hline \multicolumn{3}{|l|}{ Family: Radiococcaceae } \\
\hline Radiococcus planktonicus J.W.G. Lund & 4.2 & 10.0 \\
\hline \multicolumn{3}{|l|}{ Family: Hydrodictyaceae } \\
\hline Monactinus simplex (Meyen) Corda & 31.3 & 34.4 \\
\hline Pediastrum boryanum (Turpin) Meneghini & 1.0 & 2.2 \\
\hline Pediastrum duplex Meyen & 54.2 & 28.9 \\
\hline Stauridium tetras (Ehrenberg) Hegewald & 5.2 & - \\
\hline \multicolumn{3}{|l|}{ Family: Oocystaceae } \\
\hline Dactylococcus infusionum Nägeli & 3.1 & - \\
\hline Nephrocytium agardhianum Nägeli & - & 2.2 \\
\hline Oocystis elliptica W. West & 2.1 & 4.4 \\
\hline Oocystis lacustris Chodat & - & 4.4 \\
\hline Oocystis pusilla Hansgirg & 1.0 & 5.6 \\
\hline Oocystis sp. & - & 4.4 \\
\hline Oonephris obesa (W. West) Fott & 1.0 & 4.4 \\
\hline Planktosphaeria gelatinosa G.M. Smith & 55.2 & 50.0 \\
\hline \multicolumn{3}{|l|}{ Family: Golenkiniaceae } \\
\hline Golenkinia paucispina W. West \& G.S. West & 3.1 & 5.6 \\
\hline Golenkinia radiata Chodat & - & 2.2 \\
\hline \multicolumn{3}{|l|}{ Family: Micractiniaceae } \\
\hline Micractinium sp. & - & 1.1 \\
\hline Phytelios viridis Frenzel & - & 4.4 \\
\hline \multicolumn{3}{|l|}{ Family: Chlorococcaceae } \\
\hline Chlorococcum infusionum (Schrank) Meneghini & 1.0 & 2.2 \\
\hline Chlorococcum minutum R.C. Starr & & 1.1 \\
\hline Tetraedron gracile (Reinsch) Hansgirg & 2.1 & - \\
\hline \multicolumn{3}{|l|}{ Family: Scenedesmaceae } \\
\hline Crucigenia fenestrata (Schmidle) Schmidle & - & 2.2 \\
\hline Crucigenia quadrata Morren & 1.0 & 2.2 \\
\hline Desmodesmus quadricauda (Turpin) Hegewald & 6.3 & 2.2 \\
\hline Scenedesmus acuminatus (Lagerheim) Chodat & - & 1.1 \\
\hline Scenedesmus bijugus (Turpin) Kützing & 1.0 & 2.2 \\
\hline Scenedesmus obliquus (Turpin) Kutzing var. dimorphus (Turpin) Hansgirg & 1.0 & - \\
\hline \multicolumn{3}{|l|}{ Family: Coelastraceae } \\
\hline Actinastrum hantzschii Lagerheim & 2.1 & 1.1 \\
\hline Coelastrum microporum Nägeli & 9.4 & 6.7 \\
\hline Coelastrum reticulatum (P.A. Dangeard) Senn & 24.0 & 35.6 \\
\hline \multicolumn{3}{|l|}{ Family: Botryococcaceae } \\
\hline Dictyosphaerium ehrenbergianum Nägeli & 2.1 & - \\
\hline Dictyosphaerium granulatum Hindák & - & 2.2 \\
\hline Dictyosphaerium pulchellum H.C. Wood & 10.4 & 25.6 \\
\hline Dictyiosphaerium sp. & - & 1.1 \\
\hline \multicolumn{3}{|l|}{ Family: Chlorellaceae } \\
\hline Ankistrodesmus falcatus (Corda) Ralfs & - & 1.1 \\
\hline Ankistrodesmus fusiformis Corda ex Korshikov & 1.0 & \\
\hline Ankistrodesmus gracilis (Reinsch) Korshikov & - & 2.2 \\
\hline
\end{tabular}

- = absence of species. 
Table 2. Continued...

\begin{tabular}{|c|c|c|}
\hline \multirow{2}{*}{ Taxa } & \multicolumn{2}{|c|}{ Reservoirs } \\
\hline & Itaparica & Xingó \\
\hline Ankistrodesmus spiralis (W.B. Turner) Lemmermann & - & 1.1 \\
\hline Ankistrodesmus sp. & 1.0 & 1.1 \\
\hline Chlorella vulgaris Beijerinck & 16.7 & 21.1 \\
\hline Kirchneriella lunaris (Kirchner) K. Möbius & 1.0 & 1.1 \\
\hline Kirchneriella obesa (G.S. West) Schmidle & 1.0 & 2.2 \\
\hline Monoraphidium arcuatum (Korshikov) Hindák & 1.0 & - \\
\hline Monoraphidium contortum (Thuret) Komàrková-Legnerová & 2.1 & 4.4 \\
\hline Pseudoquadrigula sp. & - & 1.1 \\
\hline Quadrigula chodatii (Tanner-Füllemann) G.M. Smith & 9.4 & 15.6 \\
\hline Quadrigula lacustris (Chodat) G.M. Smith & - & 4.4 \\
\hline \multicolumn{3}{|l|}{ Order: Tetrasporales } \\
\hline \multicolumn{3}{|l|}{ Family: Tetrasporaceae } \\
\hline Tetraspora $\mathrm{sp}$. & - & 1.1 \\
\hline \multicolumn{3}{|l|}{ Order: Palmellaceae } \\
\hline Sphaerocystis schroeteri Chodat & 21.9 & 45.6 \\
\hline \multicolumn{3}{|l|}{ Order: Oedogoniales } \\
\hline \multicolumn{3}{|l|}{ Family: Oedogoniaceae } \\
\hline Oedogonium sp. & 1.0 & - \\
\hline \multicolumn{3}{|l|}{ Order: Volvocales } \\
\hline \multicolumn{3}{|l|}{ Family: Volvocaceae } \\
\hline Eudorina elegans Ehrenberg & 5.2 & 8.9 \\
\hline Eudorina sp. & - & 2.2 \\
\hline Volvox sp. & - & 2.2 \\
\hline \multicolumn{3}{|l|}{ Zygnematophyceae } \\
\hline \multicolumn{3}{|l|}{ Order: Zygnematales } \\
\hline \multicolumn{3}{|l|}{ Family: Zygnemataceae } \\
\hline Mougeotia sp. & 2.1 & 8.9 \\
\hline Spirogyra sp. & 2.1 & 1.1 \\
\hline \multicolumn{3}{|l|}{ Family: Closteriaceae } \\
\hline Closterium sp. & 2.1 & - \\
\hline \multicolumn{3}{|l|}{ Order: Desmidiales } \\
\hline \multicolumn{3}{|l|}{ Family: Desmidiaceae } \\
\hline Cosmarium margaritatum (P. Lundell) J. Roy \& Bisset & - & 6.7 \\
\hline Cosmarium sp. & 5.2 & 4.4 \\
\hline Desmidium sp. & 9.4 & 7.8 \\
\hline Euastrum sp. & 1.0 & - \\
\hline Onychonema laeve Nordstedt & 9.4 & 8.9 \\
\hline Pleurotaenium trabecula Nägeli & 1.0 & - \\
\hline Staurastrum angulatum West & 1.0 & - \\
\hline Staurastrum cuspidatum Brébisson in Ralfs & 1.0 & - \\
\hline Staurastrum gracile Ralfs ex Ralfs & 1.0 & 2.2 \\
\hline Staurastrum leptocladum L.N. Johnson & 17.7 & 2.2 \\
\hline Staurastrum leptacanthum Nordstedt & - & 25.6 \\
\hline Staurastrum pseudosebaldi Wille & - & 2.2 \\
\hline Staurastrum rotula Nordstedt & 15.6 & 36.7 \\
\hline Staurastrum tetracerum Ralfs & - & 2.2 \\
\hline Staurastrum triangularis (Lagerheim) Teiling & 1.0 & - \\
\hline Staurastrum sp. 1 & 8.3 & 31.1 \\
\hline Staurastrum sp. 2 & - & 3.3 \\
\hline
\end{tabular}

- = absence of species. 
Table 2. Continued...

\begin{tabular}{lcc}
\multicolumn{1}{c}{ Taxa } & \multicolumn{2}{c}{ Reservoirs } \\
\cline { 2 - 3 } & Itaparica & Xingó \\
\hline Staurodesmus cuspidatus (Brébisson) Teiling & 1.0 & - \\
Staurodesmus mamillatus (Nordstedt) Teiling & 1.0 & - \\
Staurodesmus subulatus (Kützing) Thomasson & - & 2.2 \\
Staurodesmus triangularis (Lagerheim) Teiling & - & 1.1 \\
Staurodesmus sp. & 5.2 & 7.8 \\
Family: Peniaceae & & \\
Gonatozygon monotaenium De Bary & 2.1 & 6.7 \\
Gonatozygon pilosum Wolle & - & 3.3 \\
Gonatozygon sp. & 1.0 & - \\
\hline
\end{tabular}

- = absence of species.

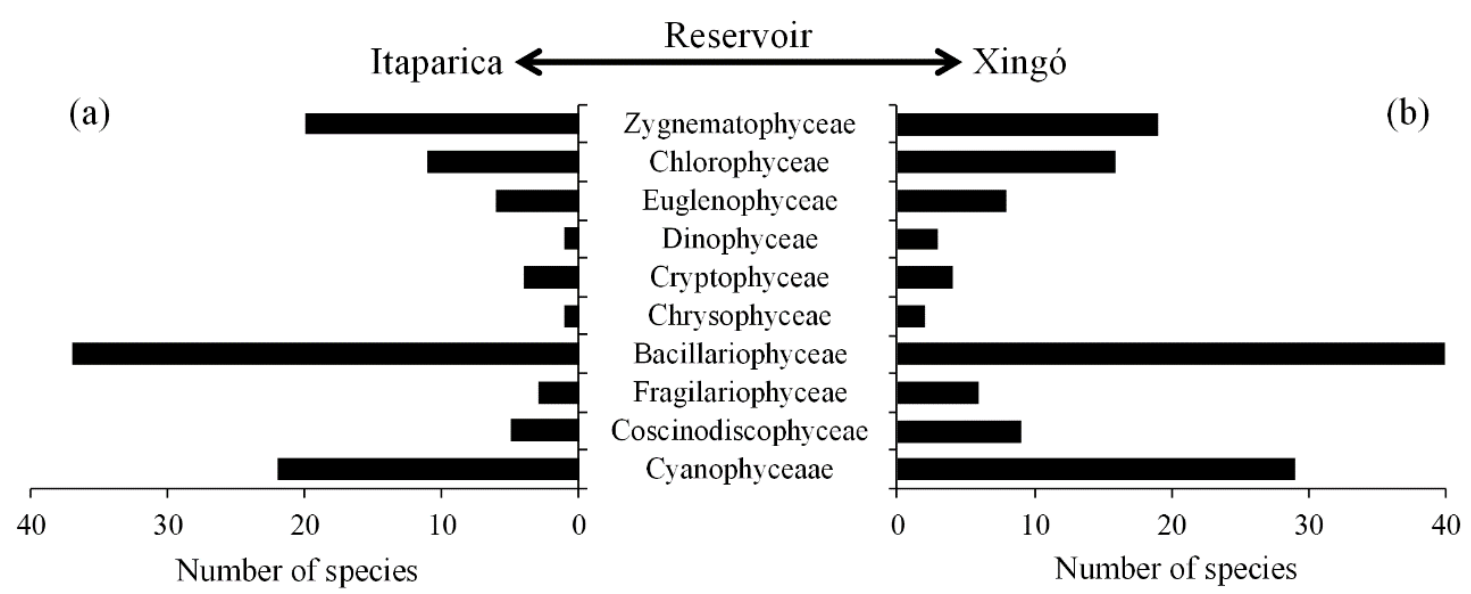

Figure 2. Species richness of phytoplankton in the (a) Itaparica and (b) Xingó Reservoirs, São Francisco River, Brazil, between December 2007 and September 2008.

cause displacement of typically periphytic organisms or those that live in the sediment to the surface layers of the water column (De León and Chalar, 2003).

Analysis of the frequency of occurrence showed that in both reservoirs studied, most species were rare and occasional (Figure 3, Table 2). There were no very frequent species in the Itaparica reservoir. However, in the frequent category, the following species were observed: the cyanobacteria Cylindrospermopsis raciborskii (Woloszynska) Seenaya \& Subba Raju, the diatoms Aulacoseira ambigua (Grunow) Simonsen and $A$. granulata (Ehrenberg) Simonsen and the green algae Pediastrum duplex Meyen and Planktosphaeria gelatinosa G.M. Smith (Table 2).

In the Xingó reservoir, Aulacoseira granulata and Fragilaria crotonensis Kitton were considered very frequent, while the cyanobacteria Cylindrospermopsis raciborskii and Geitlerinema amphibium (C. Agardh) Anagnostidis, the diatoms Aulacoseira ambigua and Urosolenia longiseta (Zacharias) Bukhtiyarova and the green algae Planktosphaeria gelatinosa and Sphaerocystis schroeteri Chodat were frequent (Table 2). The very frequent and/or frequent species in both reservoirs, C. raciborskii, A. granulata,

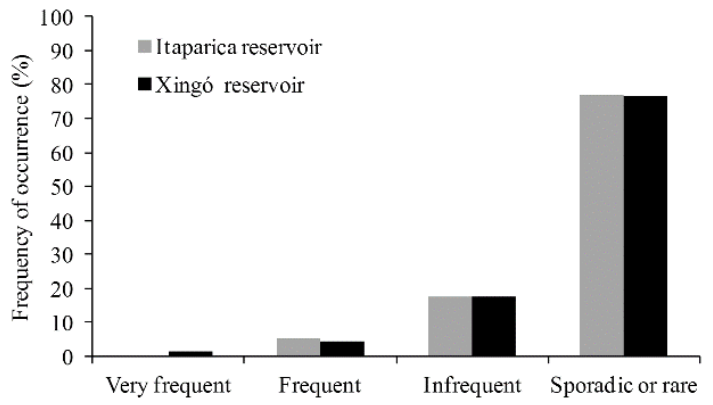

Figure 3. Frequency of occurrence of phytoplankton in the Itaparica and Xingó Reservoirs, São Francisco River, Brazil, between December 2007 and September 2008.

A. ambigua and $P$. gelatinosa, are cosmopolitan, widely distributed in freshwater ecosystems. C. raciborskii is widely studied since it forms dense blooms in water bodies and has the potential to produce toxins that are harmful to humans and other animals. It presents multiple adaptive strategies that enable it to tolerate wide ranges of environmental conditions, such as resistance to herbivory, 
tolerance to low light intensity, ability to migrate in the water column, ability to store and use intracellular stores, and the ability to fix atmospheric nitrogen (Ogawa and Carr, 1969; Bittencourt-Oliveira et al., 2011).

The occurrence of C. raciborskii has been observed in eutrophic environments with high temperature (Padisák and Reynolds, 1998), reduced water transparency (Pádisak and Reynolds, 1998; Bouvy et al., 1999; Mischke and Nixdorf, 2003) and low N/ P ratio (Briand et al., 2002).

Centric diatoms of the genus Aulacoseira inhabit mixed environments with high trophic levels (Reynolds et al., 2002 and Pádisák et al., 2009). The genus includes the species Aulacoseira granulate, one of the few diatoms that form blooms in hypereutrophic freshwater environments (Vieira et al., 2008). The cylindrical cells of these organisms are linked together to form long filaments that exhibit high sedimentation rate due to the frustule which is denser than the surrounding water (Wolin and Duthie, 1999). Therefore, the presence of this diatom in the light layers of the water column often depends on the occurrence of events that keep the mixture in suspension (Siqueiros-Beltrones, 1988; De León and Chalar, 2003).

$P$. gelatinosa occurs in environments with a clear epilimnion, low nutrient concentration and high turbidity (Reynolds et al., 2002; Padisák et al., 2009). According to Chamixaes (1990), green algae are commonly found in places with lower degrees of eutrophication.

Although the taxonomic composition in the Itaparica and Xingó reservoirs is similar, there was a difference in the occurrence of frequent and very frequent species, suggesting differences, in the environmental conditions of each reservoir, that may be related to the actual morphometry and hydrodynamics of the reservoirs, as well as to the presence of vegetation and human activities on the banks.

\section{Acknowledgements}

The authors wish to express their thanks to CHESF (Companhia Hidro Elétrica do São Francisco) and Conselho Nacional de Desenvolvimento Científico e Tecnológico (CNPq) for financial support (Proc. 301715/2008-4, 302068/2011-2 and 471603/2012-0).

\section{References}

Agência Nacional das Águas - ANA, 2013. Região Hidrográfica do São Francisco. Águas que contribuem para o desenvolvimento de 521 municípios. ANA. Available in: <http://www2.ana.gov.br/ Paginas/portais/bacias/SaoFrancisco.aspx>. Access: 01 July 2013.

ATICI, T. and OBALI, O., 2006. Seasonal variation of phytoplankton and value of chlorophyll a in the Sariyar Dam Reservoir Ankara, Turkey. Turkish Journal of Botany, vol. 30, p. 349-357.

ATICI, T., 2002. Nineteen new records from Sarýyar Dam Reservoir phytoplankton for Turkish freshwater algae. Turkish Journal of Botany, vol. 26, p. 485-490.

BITTENCOURT-OLIVEIRA, MC., MOURA, AN., HEREMAN, TC. and DANTAS, EW., 2011. Increase in straight and coiled Cylindrospermopsis raciborskii (Cyanobacteria) populations under conditions of thermal de-stratification in a shallow Tropical Reservoir. Journal of Water Resource and Protection, vol. 3, no. 4, p. 245-252. http://dx.doi.org/10.4236/jwarp.2011.34031.

BORGES, PAF., TRAIN, S. and RODRIGUES, LC., 2008. Spatial and temporal variation of phytoplankton in two subtropical Brazilian reservoirs. Hydrobiologia, vol. 607, no. 1, p. 63-74. http://dx.doi.org/10.1007/s10750-008-9367-3.

BOUVY, M., MOLICA, R., OLIVEIRA, S., MARINHO, M. and BEKER, B., 1999. Dynamics of a toxic cyanobacterial bloom (Cylindrospermopsis raciborskii) in a shallow reservoir in the semi-arid region of northern Brazil. Aquatic Microbial Ecology, vol. 20, p. 285-297. http://dx.doi.org/10.3354/ame020285.

Brasil. Ministério de Minas e Energia - MME, 2013. CHESF. MME. Available from: <http://www.chesf.gov.br/portal/page/ portal/chesf_portal/paginas/sistema_chesf/sistema_chesf_geracao/ conteiner_geracao?p_name $=8$ A2EEABD3BE1D002E0430A80 3301D002>. Access in: 01 July 2013.

BRIAND, JF., ROBILLOT, C., QUIBLIER-LLOBÉRAS, C., HUMBERT, JF., COUTÉ, A. and BERNARD, C., 2002. Environmental context of Cylindrospermopsis raciborskii (Cyanobacteria) blooms in a shallow pond in France. Water Research, vol. 36, no. 13, p. 3183-3192. http://dx.doi.org/10.1016/S0043-1354(02)00016-7. PMid:12188114.

CALIJURI, MC., SANTOS, ACA. and JATI, S., 2002. Temporal changes in the phytoplankton community structure in a tropical and eutrophic reservoir (Barra Bonita, SP - Brazil). Journal of Plankton Research, vol. 24, no. 7, p. 617-634. http://dx.doi. org/10.1093/plankt/24.7.617.

CARR, JM., HERGENRADER, GL. and TROELSTRUP JUNIOR, NH., 1986. A simple, inexpensive method for cleaning diatoms. Transactions of the American Microscopical Society, vol. 105, no. 2, p. 152-157. http://dx.doi.org/10.2307/3226387.

ÇETIN, AK. and ŞEN, B., 1998. Diatoms (Bacillariophyta) in the phytoplankton of Keban reservoir and their seasonal variations. Turkish Journal of Botany, vol. 22, p. 25-33.

CHAMIXAES, CBCB., 1990. Ficoflórula do Açude de Apipucos (Recife-PE). Brazilian Journal of Biology $=$ Revista Brasileira de Biologia, vol. 50, no. 1, p. 45-60.

CHELLAPPA, NT., CÂMARA, FRA. and ROCHA, O., 2009. Phytoplankton community: indicator of water quality in the Armando Ribeiro Gonçalves reservoir and Pataxó channel, Rio Grande do Norte, Brazil. Brazilian Journal of Biology $=$ Revista Brasileira de Biologia, vol. 69, no. 2, p. 241-251. http://dx.doi. org/10.1590/S1519-69842009000200003. PMid:19675924.

DE LEÓN, L. and CHALAR, G., 2003. Abundancia y diversidad del fitoplancton en el Embalse de Salto Grande (ArgentinaUruguay). Ciclo estacional y distribución espacial. Limnetica, vol. 22 , no. 1-2, p. 103-113.

FELISBERTO, SA. and RODRIGUES, L., 2010. Periphytic algal community in artificial and natural substratum in a tributary of the Rosana reservoir (Corvo Stream, Paraná State, Brazil). Acta Scientiarum. Biological Sciences, vol. 32, no. 4, p. 373-385. http:// dx.doi.org/10.4025/actascibiolsci.v32i4.4627.

FONTANA, L. and BICUDO, DC., 2009. Diatomáceas (Bacillariophyceae) de sedimentos superficiais dos reservatórios em cascata do Rio Paranapanema (SP/PR, Brasil): Coscinodiscophyceae e Fragilariophyceae. Hoehnea, vol. 36, no. 3, p. 375-386. http:// dx.doi.org/10.1590/S2236-89062009000300001. 
FRANCA, LMB. and COIMBRA, MML., 1998. Fitoplâncton coletado num período de 24 horas no Reservatório de Itaparica (PE/BA). Caderno Ômega, vol. 4, p. 69-83.

FRANCESCHINI, IM., BURLIGA, AL., REVIERS, B., PRADO, JF. and RÉZIG, SH., 2010. Algas: uma abordagem filogenética, taxonômica e ecológica. Porto Alegre: Artmed. 332 p.

HUSZAR, VLM., SILVA, LHS., MARINHO, M., DOMINGOS, P. and SANT'ANNA, CL., 2000. Cyanoprokaryote assemblages in eight produtive tropical Brazilian waters. Hydrobiologia, vol. 424, no. 1-3, p. 67-77. http://dx.doi.org/10.1023/A:1003996710416.

KELLY, VJ., 2001. Influence of reservoirs on solute transport: a regional-scale approach. Hydrological Processes, vol. 15, no. 7, p. 1227-1249. http://dx.doi.org/10.1002/hyp.211.

KOMÁREK, J. and ANAGNOSTIDIS, K., 2000. Cyanoprokaryota. 1. Teil: chroococcales. In BÜDEL, B., KRIENITZ, L., GÄRTNER, G. and SCHAGERLV, M. (Eds.). Süsswasserflora Von Mitteleuropa 19/1. Heidelberg: Elsevier/Spektrum. p. 1-548 p.

KOMÁREK, J. and ANAGNOSTIDIS, K., 2005. Cyanoprokaryota 2. Teil/ 2nd Part: oscillatoriales. In BÜDEL, B., KRIENITZ, L., GÄRTNER, G. and SCHAGERLV, M. (Eds.). Süsswasserflora Von Mitteleuropa 19/2. Heidelberg: Elsevier/Spektrum. p. 1-759.

LI, Z., WANG, S., GUO, J., FANG, F., GAO, X. and LONG, M., 2012. Responses of phytoplankton diversity to physical disturbance under manual operation in a large reservoir, China. Hydrobiologia, vol. 684, no. 1, p. 45-56. http://dx.doi.org/10.1007/ s10750-011-0963-2.

MAGILLIGAN, FJ. and NISLOW, KH., 2005. Changes in hydrologic regime by dams. Geomorphology, vol. 71, no. 1-2, p. 61-78. http://dx.doi.org/10.1016/j.geomorph.2004.08.017.

MALAIWAN, T. and PEERAPORNPISAL, Y., 2009. Diversity of Phytoplankton and water quality in the reservoir of Nam Ngum Dam, Lao PDR. KKU Science Journal, vol. 37, supplement, p. 42-49.

MATTEUCCI, SD. and COLMA, A., 1982. Metodologia para el estudio de la vegetacion. Washington: The General Secretarial of the Organization of American States. 168 p. Série de Biologia - Monografia, no. 22.

MELO-MAGALHÃES, EM., LIRA, MCA., COSTA, FJCB. and SILVA, VB., 2000. Diversidade e variação espaço-temporal do fitoplâncton no reservatório da Usina Hidroelétrica de Xingó. Boletim de Estudos de Ciências do Mar, vol. 11, p. 1-14.

MISCHKE, U. and NIXDORF, B., 2003. Equilibrium phase conditions in shallow German lakes: how Cyanoprocaryota species establish a steady state phase in late summer. Hydrobiologia, vol. 502, no. $1-3$, p. 123-132. http://dx.doi.org/10.1023/ B:HYDR.0000004275.81490.92

NOGUEIRA, MG., 2000. Phytoplankton composition, dominance and abundance as indicators of environmental compartmentalization in Jurumirim Reservoir (Paranapanema River), São Paulo, Brazil. Hydrobiologia, vol. 431, no. 2-3, p. 115-128. http://dx.doi. org/10.1023/A:1003769408757.

NOGUEIRA, MG., FERRAREZE, M., MOREIRA, ML. and GOUVÊA, RM., 2010. Phytoplankton assemblages in a reservoir cascade of a large tropical - subtropical river (SE, Brazil). Brazilian Journal of Biology = Revista Brasileira de Biologia, vol. 70, no. 3, supplement, p. 781-793. http://dx.doi.org/10.1590/ S1519-69842010000400009. PMid:21085783.
OGAWA, RE. and CARR, NG., 1969. The influence of nitrogen on heterocyst production in blue-green algae. Limnology and Oceanography, vol. 14, no. 3, p. 342-351. http://dx.doi.org/10.4319/ lo.1969.14.3.0342

OLIVEIRA, MT., ROCHA, O. and PERET, AC., 2011. Structure of the phytoplankton community in the Cachoeira Dourada reservoir (GO/MG), Brazil. Brazilian Journal of Biology $=$ Revista Brasileira de Biologia, vol. 71, no. 3, p. 587-600. PMid:21881782.

PADISÁK, J. and REYNOLDS, CS., 1998. Selection of phytoplankton associations in Lake Balaton, Hungary, in response to eutrophication and restoration measures, with special reference to the cyanoprokaryotes. Hydrobiologia, vol. 384, no. 1-3, p. 4153. http://dx.doi.org/10.1023/A:1003255529403.

PADISÁK, J., CROSSETTI, LO. and NASELLI-FLORES, L., 2009. Use and misuse in the application of the phytoplankton functional classification: a critical review with updates. Hydrobiologia, vol. 621, no. 1, p. 1-19. http://dx.doi.org/10.1007/s10750-008-9645-0.

PÉREZ, MC., BONILLA, S. and MARTÍNEZ, G., 1999. Phytoplankton community of a polymictic reservoir, La Plata River basin, Uruguay. Brazilian Journal of Biology $=$ Revista Brasileira de Biologia, vol. 59, no. 4, p. 535-541. http://dx.doi. org/10.1590/S0034-71081999000400002. PMid:23505641.

POPOVSKAYA, GI., FIRSOVA, AD., BESSUDOVA, AY., SAKIRKO, MV., SUTURIN, AN. and LIKHOSHWAY, YV., 2012. Phytoplankton of the Irkutsk Reservoir as an indicator of water quality. International Journal of Oceanography and Hydrobiology, vol. 41, no. 2, p. 29-38. http://dx.doi.org/10.2478/ s13545-012-0014-2.

PRINGLE, C., 2003. What is hydrologic connectivity and why is it ecologically important? Hydrological Processes, vol. 17, no. 13, p. 2685-2689. http://dx.doi.org/10.1002/hyp.5145.

REVIERS, B., 2006. Biologia e Filogenia das Algas. Porto Alegre: Artmed. 280 p.

REYNOLDS, CS., HUSZAR, V., KRUK, C., NASELLI-FLORES, L. and MELO, S., 2002. Towards a functional classification of the freshwater phytoplankton. Journal of Plankton Research, vol. 24, no. 5, p. 417-428. http://dx.doi.org/10.1093/plankt/24.5.417.

ROUND, FE., CRAWFORD, RM. and MANN, DG., 1990. The Diatoms. Biological \& morphology of the genera. Cambridge: Cambridge University Press. 747 p.

SILVA, AM., LUDWIG, TAV., TREMARIN, PI. and VERCELLINO, IS., 2010. Diatomáceas perifíticas em um sistema eutrófico brasileiro (Reservatório do Iraí, estado do Paraná). Acta Botanica Brasílica, vol. 24, no. 4, p. 997-1016. http://dx.doi.org/10.1590/ S0102-33062010000400015.

SILVA, CA., TRAIN, S. and RODRIGUES, LC., 2005. Phytoplankton assemblages in a Brazilian subtropical cascading reservoir system. Hydrobiologia, vol. 537, no. 1-3, p. 99-109. http://dx.doi.org/10.1007/s10750-004-2552-0.

SILVA, SRVF. and CECY, IIT., 2004. Desmídias (Zygnemaphyceae da área de abrangência da Usina Hidrelétrica de Salto Caxias, Paraná, Brasil, I: Gênero Cosmarium. Iheringia Série Botânica., vol. 59 , no. 1, p. 13-26.

SIQUEIROS-BELTRONES, DA., 1988. Diatomeas bentônicas de la Laguna Figueiroa, Baja California. Ciencias Marinas, vol. 14 , no. 2 , p. $85-112$. 
TUNDISI, JG. and MATSUMURA-TUNDISI, T., 2003. Integration of research and management in optimizing multiple uses of reservoirs: the experience of South America and Brazilian case studies. Hydrobiologia, vol. 500, no. 1-3, p. 231-242. http:// dx.doi.org/10.1023/A:1024617102056.

TUNDISI, JG., MATSUMURA-TUNDISI, T. and ROCHA, O., 2002. Ecossistemas de águas interiors. In REBOUÇAS, AC., BRAGA, B. and TUNDISI, JG. (Eds.). Águas doces no Brasil: capital ecológico, uso e conservação. 2nd ed. São Paulo: Escrituras. p. 153-194.

VAN DEN HOEK, C., MANN, DG. and JAHNS, HM., 1995. Algae: an introduction to phycology. Cambridge: Cambridge University Press. 640 p.
VIEIRA, AAH., ORTOLANO, PIC., GIROLDO, D., OLIVEIRA, MJD., BITTAR, TB., LOMBARDI, AT., SARTORI, AL. and PAULSEN, BS., 2008. Role of hydrophobic extracellular polysaccharide of Aulacoseira granulate (Bacillariophyceae) on aggregate formation in a turbulent and hypereutrophic reservoir. Limnology and Oceanography, vol. 53, no. 5, p. 1887-1899. http:// dx.doi.org/10.4319/1o.2008.53.5.1887.

WETZEL, RG., 1993. Limnologia. Lisboa: Fundação Calouste Gulbenkian. 919 p.

WOLIN, JA. and DUTHIE, H., 1999. Diatoms as indicators of water level change in freshwater lakes. In STOERMER, EF. and SMOL, JP. (Eds.). The diatoms: applications for the environmental and earth sciences. Cambridge: Cambridge University Press. p. 183-202. 